\title{
IMblaze 370: lessons learned and future strategies in colorectal cancer treatment
}

\author{
Mark McGregor ${ }^{1}$, Timothy J. Price ${ }^{1,2}$ \\ ${ }^{1}$ Department of Medical Oncology, The Queen Elizabeth Hospital, Woodville, Australia; ${ }^{2}$ University of Adelaide, School of Medicine, Adelaide, \\ Australia \\ Correspondence to: Timothy J. Price. Department of Medical Oncology, The Queen Elizabeth Hospital, 28 Woodville Road, Woodville 5011, \\ Australia. Email: timothy.price@sa.gov.au. \\ Provenance: This is an invited article commissioned by the Section Editor Dr. Zheng-Wei Leng (Deputy Director/Associate Professor, Department of \\ Hepatobiliary Surgery, the Affiliated Hospital of North Sichuan Medical University, Nanchong, China). \\ Comment on: Eng C, Kim TW, Bendell J, et al. Atezolizumab with or without cobimetinib versus regorafenib in previously treated metastatic \\ colorectal cancer (IMblaze370): a multicentre, open-label, phase 3, randomised, controlled trial. Lancet Oncol 2019;20:849-61.
}

Submitted Aug 23, 2019. Accepted for publication Aug 29, 2019.

doi: 10.21037/atm.2019.08.119

View this article at: http://dx.doi.org/10.21037/atm.2019.08.119

\section{Introduction}

Despite recent advances in systemic treatment of metastatic colorectal cancer (mCRC) where surgical resection is not possible, 5 -year overall survival (OS) rates are still poor. Beyond the standard first and secondline combinations of fluoropyrimidine, oxaliplatin and irinotecan with VEGF and EGFR inhibitors where appropriate, there has been little success in finding additional systemic treatment options to significantly prolong survival. With the exception of mismatch repair (MMR) deficient colorectal cancers, checkpoint inhibitor immunotherapy has not yielded the same promising results as it has in a number of other malignancies.

For the majority of patients, current standards of care in $3^{\text {rd }}$ line and beyond are regorafenib or TAS-102, however these have shown only very modest improvements over best supportive care $(1,2)$. It is in this pre-treated setting of mCRC that Eng and colleagues explored the use of atezolizumab, an immune checkpoint inhibitor targeting programmed cell death-ligand 1 (PD-L1), with or without the MEK inhibitor cobimetinib, versus regorafenib in the IMblaze 370 trial (3). This trial was designed based on phase 1 results of combined atezolizumab \& cobimetinib in refractory mCRC (4), in the broader context of trying to improve responses to immunotherapy of mCRC, in particular in those without MMR deficiency. Regorafenib was selected for the control group as a suitable standard of care comparator in those progressing on standard firstand second-line therapies, despite it only having marginal survival benefit (1.4 months) over best supportive care in the CORRECT trial of heavily pre-treated mCRC patients (2).

\section{Summary of results}

In 363 enrolled mCRC patients (92\% with confirmed microsatellite stable (MSS) disease), who had received at least 2 prior treatments, the study's primary endpoint was not met, with median OS not differing between atezolizumab + cobimetinib $(\mathrm{A}+\mathrm{C}, 8.87$ months $)$ and regorafenib (R, 8.51 months, HR 1.00 (95\% CI, 0.73-1.38)]; or between atezolizumab monotherapy (A, 7.10 months) and regorafenib [R, 8.51 months, HR 1.19 (95\% CI, 0.831.71)]. There was also no progression free survival (PFS) benefit of atezolizumab +/- cobimetinib over regorafenib, with regorafenib actually showing a trend to superiority. Objective response rates (ORR) were $3 \%, 2 \%$ and $2 \%$ in $\mathrm{A}+\mathrm{C}, \mathrm{A}$ and $\mathrm{R}$ respectively. Treatment efficacy (OS or PFS) did not differ by subgroups such as RAS mutation status and PD-L1 expression.

Microsatellite instability (MSI)/deficient mismatch repair (dMMR) represented less than $2 \%$ of enrolled patients. None of these MSI-H/dMMR patients were assigned to the regorafenib group, making it impossible to form any meaningful conclusions of comparative treatment effect in this subgroup. There was, as expected, activity of checkpoint 
inhibition in MSI-H/dMMR subgroup, with responses observed in 2 of the 3 MSI-H patients with atezolizumab \& cobimetinib and 1 of 3 MSI-H patients in atezolizumab monotherapy group. PD-L1 status did impact on ORR (higher in atezolizumab-containing groups), but not on OS or PFS. Overall grade 3/4 toxicities were similar between $\mathrm{A}+\mathrm{C} \&$ regorafenib $(60 \%$ vs. $58 \%)$, with addition of cobimetinib almost doubling the rate of grade $3 / 4$ toxicity seen with atezolizumab alone ( $31 \%$ grade $3 / 4$ toxicity).

\section{Discussion and commentary}

To examine the reasons behind this negative study, we need to look at the population and methodology used in the trial and then assess if the underlying hypothesis behind this trial was robust. The patients recruited were those that progressed on at least two lines of therapy. Of note, only $26 \%$ of patients included had received more than 3 lines of previous treatment, compared to $48 \%$ of patients in the CORRECT trial which led to regulatory approval for regorafenib in pre-treated colorectal cancer (2). This less pre-treated population may have played some role in the higher OS seen in the regorafenib control group of IMBlaze 370 compared to patients receiving the same treatment in CORRECT (median OS 8.5 vs. 6.4 months). The better than expected outcome of the control group potentially negated the chance of finding a meaningful survival benefit of this experimental combination. It does, however suggest some underlying biological activity of the atezolizumab \& cobimetinib combination, given the hazard ratio of 1.0 for OS when compared to regorafenib, a treatment already shown to have activity in a more pre-treated population than seen in this trial.

The signal of increased activity with checkpoint inhibition in the dMMR subgroup is consistent with numerous studies demonstrating activity of checkpoint inhibition almost exclusively in MMR deficiency. A study by Le \& colleagues using pembrolizumab in pretreated metastatic malignancies with MMR deficiency, demonstrated ORR of $52 \%$ in colorectal cancers with a 2 -year PFS of $53 \%$, compared to $0 \%$ ORR in pMMR colorectal cancers $(5,6)$. Checkmate 142 confirmed these findings with significant activity in $\mathrm{d} M M \mathrm{R}$ colorectal cancers seen with both single agent nivolumab (ORR 31\%) and combination ipilimumab \& nivolumab (ORR 55\%) in a similarly pre-treated patient population. Many of those responders continued to have a durable prolonged response. Once again, this benefit was confined to the dMMR group with no responses seen in pMMR in the early phase of this trial, however the benefit appeared to persist regardless of RAS/BRAF or PD-L1 status $(7,8)$. The specific benefit of checkpoint inhibitors in this group of patients has been hypothesized to be due to the hypermutated nature of tumours deficient in MMR, with multiple neoantigens generated that have the potential to be recognized as nonself by the host's immune system, leading to an "inflamed" immune phenotype with intense lymphocytic infiltrate in the tumour micro-environment (TME) (9). The majority of pMMR colorectal cancers have a far lower tumour mutational burden leading to a less immunogenic TME, with phenotypes either described as "immune desert" (absent T-cells) or "immune-excluded" (T-cells at periphery of tumour but not infiltrating) $(9,10)$. There also appears to be marked intra and inter-tumoural heterogeneity in this population, leading to formation of subclonal, rather than clonal neoantigens, limiting T-cell response to immunotherapy $(11,12)$.

The PD-L1 inhibitor, atezolizumab, would therefore be expected to have activity in the $\mathrm{dMMR}$ group of patients. However, a disappointing aspect of this particular trial is that the patient numbers are far too small to appreciate if cobimetinib adds to the immunogenic response in this subgroup [atezolizumab + cobimetinib MSI-H ORR 2/3 (66\%) vs. atezolizumab MSI-H ORR 1/3 (33\%)], who would be inherently more sensitive to checkpoint inhibitor monotherapy than the overall population of majority pMMR colorectal cancers. It is therefore difficult to speculate whether this strategy should be re-attempted in more targeted population of dMMR colorectal cancers, with no real pre-existing data for this particular subgroup, other than the combined checkpoint inhibition having benefit over monotherapy $(7,8)$.

\section{Inbibition of MEK as a therapeutic strategy}

The hypothesis behind the addition of cobimetinib to checkpoint inhibitor immunotherapy arises from the search for strategies to convert immune-resistant $\mathrm{pMMR}$ colorectal cancer, into more immunogenic tumours with favourable properties to elicit response to immune checkpoint inhibitors. Preclinical data had suggested that signalling through the mitogen-activated protein kinase (MAPK) pathway contributed to immune escape through the upregulation of immunosuppressive cytokines and downregulation of major histocompatibility complex class 1 (MHC1). Furthermore, blockade of this pathway through 
the use of a MEK1 \& MEK2 inhibitor, cobimetinib, was seen to augment anti-tumour activity of PD-1 inhibitors in KRAS mutant, pMMR cell lines. Synergistic tumour regression was seen when the two agents were combined, through increased T-cell infiltration and increased MHC1 expression on tumours (13). When taken to phase 1 trial in refractory colorectal cancer, the combination of atezolizumab and cobimetinib initially yielded objective response in 4 out of 23 patients (17\%), with 3 of the 4 confirmed to have pMMR tumours (4). Updated results from the expanded cohort of 84 patients (66 patients with $\geq 5$ prior treatments), reported after initiation of phase 3 trial, yielded a confirmed ORR of $7 \%$ with a median PFS of 1.9 months (14). Of note, the median OS reported was 10 months, substantially greater than expected for standard of care in this population and greater than what was seen in the less heavily pre-treated population of IMBlaze 370 (8.9 months). Responses were seen in either pMMR/MSIlow/MSS disease or MSI unknown but did not correlate to baseline PD-L1 expression. Serial biopsy cohort were noted to have the upregulated MHC1 expression and CD8 T-cell infiltration predicted by the pre-clinical data.

A number of questions arise from this promising preclinical and early phase data not translating to similar success in IMBlaze 370. Firstly is the underlying biological rationale and hypothesis sound? It may have had backing from some pre-clinical studies, however there is controversy as to the true role that the MAPK pathway, and its inhibition, may play in tumour biology. MEK is thought to be required for later stages of T-cell differentiation, such as generation of memory cells or cytotoxic T-cell activity, which may be an important population for immunotherapy efficacy, with MEK inhibition potentially suppressing this population of effector cells $(15,16)$. Furthermore, MEK inhibition has been shown in other pre-clinical studies to suppress T-cell proliferation, cytokine production and antigen expansion and processing through dendritic cells (17).

\section{Trial design \& the search for biomarkers}

With this conflicting pre-clinical data at least creating doubt as to the biological rationale and plausibility of MEK inhibition enhancing immune response, a bigger question arises: Was the leap too great to proceed straight from a phase 1 trial with modest results, to a large phase 3 trial, with an active comparator, in an unselected population of metastatic CRC patients, comprising of a majority of notoriously immunotherapy-resistant pMMR tumours?
There did appear to be a small benefit of the addition of cobimetinib to atezolizumab over atezolizumab alone (median OS 8.87 vs. 7.10 months, 12 -month OS 38.5\% vs. $27.2 \%$ ), however the trial statistical design did not see these groups directly compared and therefore this observed difference is purely hypothesis generating. As such, it remains unclear if this combination should be pursued in a separate trial with a direct comparison between treatments, in either unselected or MSI high colorectal cancers, where a true difference may be able to be uncovered between groups. A smaller trial directly comparing these two groups, or comparing to best supportive care in the setting of regorafenib failure, may have been a better initial strategy.

A further limitation to this study is the lack of ability to define any other biomarker-based subgroups which may benefit from checkpoint inhibitor-based treatment, beyond the well-known groups of RAS/BRAF mutant $v$ s. wildtype, PD-L1 expression and MMR status. A strategy using either tumour or liquid biopsies to identify a population more responsive to atezolizumab and cobimetinib combination was not pursued. A successful example of this strategy was seen in a trial of Sym004 in anti-EGFR refractory colorectal cancer patients. In this trial, the study drug was not shown to be superior to investigator's choice treatment in intent-to-treat population, however liquid biopsies through circulating tumour DNA identified a molecular signature subgroup with markedly improved survival of study drug over controls. This provided a rationale for a prospective clinical trial to validate these findings (18). With over $90 \%$ of participants in IMBlaze 370 having known $\mathrm{pMMR}$ disease, a group with limited activity to checkpoint inhibition, a trial exploring the activity of immunotherapy ideally needs to be identifying potential biomarkers, patient factors or immune phenotypes of the tumours to find commonalities in any of the patients that may benefit. It is already known that there are some pMMR tumours with phenotypes similar to dMMR cancers (higher numbers of infiltrating T-cells and inflammatory cytokines) that have improved prognosis (19). Therefore molecular profiling using tissue or liquid biopsies to identify genes encoding a more "dMMR-like phenotype" in pMMR cancers may potentially identify subgroups that may either benefit from PD-L1 inhibition alone or perhaps need the addition of an agent such as cobimetinib. This is particularly essential due to the high rate of heterogeneity within the $\mathrm{pMMR}$ population. Recently presented data analyzing 63 core biopsies from pMMR colorectal cancer liver metastases revealed $27 \%$ immune-reactive tumours, based on immune 
gene expression profiling, with high rates of tumourinfiltrating lymphocytes noted in immune-reactive tumours. Interestingly however, there was no correlation between number of somatic mutations and immune-responsiveness. These biopsies were also able to isolate tumour specific immune checkpoints and their ligands in the immunereactive cancers, allowing the personalized targeting through checkpoint inhibitors more biologically relevant than PD-1 and CTLA4 (20). This more tailored approach likely represents the future for further trial design.

\section{Conclusions and future strategies}

It remains possible that adding a targeted agent to checkpoint inhibition to restore immunogenicity of a tumour such as pMMR colorectal cancer is an ineffective strategy, with similar disappointing results seen with bevacizumab added to PD-L1 in the MODUL study (21). However there have been some promising results supporting ongoing investigation of anti-angiogenic combinations, with a Japanese phase 1 trial combining regorafenib and nivolumab yielding an ORR of $29 \%$ in MSS colorectal cancer patients (22). Many trials continue to pursue similar strategies to enhance or convert $\mathrm{pMMR}$ colorectal cancer into a tumour that can be recognized and eliminated through immune modulation. The MODULATE study is one of these studies, enrolling patients with $\mathrm{pMMR}$ colorectal cancers to receive checkpoint inhibitor therapy in combination with either a vascular disrupting agent, enhancing efflux of tumour neoantigens to the TME or an inhibitor of STAT3, a transcription factor involved in immune suppression of the (TME). This study will also use serial tumour and liquid biopsies to evaluate selected biomarkers related to immunomodulation and to potentially identify molecular subgroups that may benefit from this treatment (23), something as noted missing in the IMblaze study.

With results from the aforementioned Japanese study, as well as recently presented results of the phase 2 CO.26 trial showing an OS benefit of combined checkpoint inhibition of durvalumab and tremelimumab over best supportive care (6.6 vs. 4.1 months, HR 0.72, $\mathrm{P}=0.07$ ) in $\mathrm{pMMR} / \mathrm{MSS}$ colorectal cancers (24), checkpoint inhibitor combination trials are likely to continue to be pursued, despite the negative results of IMBlaze 370. However, lessons can be learned from this trial beyond pursuing alternate strategies to MEK inhibition. There is a need to first show benefit of the combination over standard checkpoint inhibition before proceeding to more ambitious study designs comparing against standard of care. There is also a need to understand that there is significant heterogeneity present in this population and that a uniform treatment strategy for all pMMR CRC's may not be appropriate, hence trials using correlational tissue and liquid biopsies for biomarker identification are required to appropriately tailor treatments. A large number of trials are actively recruiting, including combinations of checkpoint inhibitors with targeted therapies, radiation and chemotherapy. With the right trial design, hopefully the best immunomodulatory strategy or combination is found in the near future.

\section{Acknowledgments}

None.

\section{Footnote}

Conflicts of Interest: The authors have no conflicts of interest to declare.

Ethical Statement: The authors are accountable for all aspects of the work in ensuring that questions related to the accuracy or integrity of any part of the work are appropriately investigated and resolved.

\section{References}

1. Mayer RJ, Van Cutsem E, Falcone A, et al. Randomized trial of TAS-102 for refractory metastatic colorectal cancer. N Engl J Med 2015;372:1909-19.

2. Grothey A, Van Cutsem E, Sobrero A, et al. Regorafenib monotherapy for previously treated metastatic colorectal cancer (CORRECT): An international, multicentre, randomised, placebo-controlled, phase 3 trial. Lancet 2013;381:303-12.

3. Eng C, Kim TW, Bendell J, et al. Atezolizumab with or without cobimetinib versus regorafenib in previously treated metastatic colorectal cancer (IMblaze370): a multicentre, open-label, phase 3, randomised, controlled trial. Lancet Oncol 2019;20:849-61.

4. Bendell JC, Kim TW, Goh BC, et al. Clinical activity and safety of cobimetinib (cobi) and atezolizumab in colorectal cancer (CRC). J Clin Oncol 2016;34:abstr 3502.

5. Le DT, Uram JN, Wang H, et al. PD-1 blockade in tumors with mismatch-repair deficiency. N Engl J Med 2015;372:2509-20.

6. Le DT, Durham JN, Smith KN, et al. Mismatch repair 
deficiency predicts response of solid tumors to PD-1 blockade. Science 2017;357:409-13.

7. Overman MJ, Kopetz S, McDermott RS, et al. Nivolumab \pm ipilimumab in treatment (tx) of patients (pts) with metastatic colorectal cancer (mCRC) with and without high microsatellite instability (MSI-H): CheckMate-142 interim results. J Clin Oncol 2016;34:abstr 3501.

8. Overman MJ, Lonardi S, Wong KYM, et al. Durable Clinical Benefit With Nivolumab Plus Ipilimumab in DNA Mismatch Repair-Deficient/Microsatellite Instability-High Metastatic Colorectal Cancer. J Clin Oncol 2018;36:773-9.

9. Le DT, Hubbard-Lucey VM, Morse MA, et al. A Blueprint to Advance Colorectal Cancer Immunotherapies. Cancer Immunol Res 2017;5:942-9.

10. Chen DS, Mellman I. Elements of cancer immunity and the cancer-immune set point. Nature 2017;541:321-30.

11. Ganesh K, Stadler ZK, Cercek A, et al. Immunotherapy in colorectal cancer: rationale, challenges and potential. Nat Rev Gastroenterol Hepatol 2019;16:361-75.

12. McGranahan N, Furness AJ, Rosenthal R, et al. Clonal neoantigens elicit $\mathrm{T}$ cell immunoreactivity and sensitivity to immune checkpoint blockade. Science 2016;351:1463-9.

13. Ebert PJR, Cheung J, Yang Y, et al. MAP Kinase Inhibition Promotes T Cell and Anti-tumor Activity in Combination with PD-L1 Checkpoint Blockade. Immunity 2016;44:609-21.

14. Bendell JC, Bang Y-J, Chee CE, et al. A phase Ib study of safety and clinical activity of atezolizumab (A) and cobimetinib (C) in patients (pts) with metastatic colorectal cancer (mCRC). J Clin Oncol 2018;36:abstr 560.

15. Djeu JY, Jiang K, Wei S. A view to a kill: signals triggering cytotoxicity. Clin Cancer Res 2002;8:636-40.

16. Liu X, Gibbons RM, Harrington SM, et al. Endogenous tumor-reactive CD8(+) T cells are differentiated effector cells expressing high levels of CD11a and PD-1 but are unable to control tumor growth. Oncoimmunology 2013;2:e23972.

17. Vella LJ, Pasam A, Dimopoulos N, et al. MEK inhibition, alone or in combination with BRAF inhibition, affects multiple functions of isolated normal human lymphocytes and dendritic cells. Cancer Immunol Res 2014;2:351-60.

18. Montagut C, Argilés G, Ciardiello F, et al. Efficacy of
Sym004 in Patients With Metastatic Colorectal Cancer With Acquired Resistance to Anti-EGFR Therapy and Molecularly Selected by Circulating Tumor DNA Analyses: A Phase 2 Randomized Clinical Trial. JAMA Oncol 2018;4:e175245.

19. Mlecnik B, Bindea G, Angell HK, et al. Integrative Analyses of Colorectal Cancer Show Immunoscore Is a Stronger Predictor of Patient Survival Than Microsatellite Instability. Immunity 2016;44:698-711.

20. Hébert S, Mathieu M, Henault D, et al. Abstract 579: Immune features and neoantigen recognition in mismatch repair-proficient colorectal cancer liver metastases. Cancer Res 2019;79:abstr 579.

21. Grothey A, Tabernero J, Arnold D, et al. Fluoropyrimidine (FP) + bevacizumab (BEV) + atezolizumab vs $\mathrm{FP} / \mathrm{BEV}$ in BRAFwt metastatic colorectal cancer (mCRC): Findings from Cohort 2 of MODUL - a multicentre, randomized trial of biomarker-driven maintenance treatment following first-line induction therapy. Ann Oncol 2018;29:abstr LBA19.

22. Fukuoka S, Hara H, Takahashi N, et al. Regorafenib plus nivolumab in patients with advanced gastric (GC) or colorectal cancer (CRC): An open-label, dose-finding, and dose-expansion phase 1b trial (REGONIVO, EPOC1603). J Clin Oncol 2019;37:abstr 2522.

23. Bethesda (MD): National Library of Medicine (US). $2000 \mathrm{Feb} 29$. Identifier NCT03647839, Modulation Of The Tumour Microenvironment Using Either Vascular Disrupting Agents or STAT3 Inhibition in Order to Synergise With PD1 Inhibition in Microsatellite Stable, Refractory Colorectal Cancer (MODULATE); 2018 Aug 27 [cited 2019 Aug 15]. Available online: http:// clinicaltrials.gov/ct2/show/NCT03647839

24. Chen EX, Jonker DJ, Loree JM, et al. CCTG CO.26: Updated analysis and impact of plasma-detected microsatellite stability (MSS) and tumor mutation burden (TMB) in a phase II trial of durvalumab (D) plus tremelimumab (T) and best supportive care (BSC) versus BSC alone in patients (pts) with refractory metastatic colorectal carcinoma (rmCRC). J Clin Oncol 2019;37:abstr 3512.
Cite this article as: McGregor M, Price TJ. IMblaze 370: lessons learned and future strategies in colorectal cancer treatment. Ann Transl Med 2019;7(21):602. doi: 10.21037/ atm.2019.08.119 